\title{
РЕЗОНАНСНЫЕ КОЛЕБАНИЯ С ПРЕДЕЛЬНОЙ АМПЛИТУДОЙ В ВИБРАЦИОНОМ ЭЛЕКТРОМАГНИТНОМ АКТИВАТОРЕ
}

\author{
Гаврилин Алексей Николаевич',
} tom-gawra|@list.ru

Боловин Евгений Владимирович', boloinev@mail.ru

Глазырин Александр Савельевич', asglazyrin@tpu.ru

\section{Кладиев Сергей Николаевич', kladiev@tpu.ru}

\author{
Полищук Владимир Иосифович \\ polischuk_vi@mail.ru \\ 1 Национальный исследовательский Томский политехнический университет, \\ Россия, 635050, г. Томск, пр. Ленина, 30. \\ ${ }^{2}$ Алтайский государственный технический университет им. И.И. Ползунова, \\ Россия, 656038, г. Барнаул, пр. Ленина, 46.
}

\begin{abstract}
Актуальность исследования обусловлена тем, что вибрационные электромагнитные активаторы являются перспективными для применения в различных технологиях, связанных с добычей и транспортировкой георесурсов, в том числе при приготовлении буровых растворов и разжижении вязких нефтепродуктов. Вибрационный электромагнитный активатор представляет собой электрическую машину возвратно-поступательного движения с якорем-активатором, образующим в жидкой обрабатываемой среде турбулентные затопленные струи. В первом попупериоде якорь-активатор притягивается к стенке за счет импульса тока в катушках, при этом в упругой пружине накапливается потенциальная энергия. Этот режим является режимом вынужденных колебаний в механической системе вибрационного электромагнитного активатора. Во втором полупериоде ток в катушках отсутствует, а якорь-активатор отталкивается от стенок за счет энергии пружины - это режим свободных колебаний в механической системе. С точки зрения улучшения энергоэффективности следует настраивать вибрационный электромагнитный активатор на резонансную частоту. Резонансная частота не является постоянной и зависит от свойств обрабатываемой жидкости. Форма вынуждающей колебания силы должна быть такой, чтобы обеспечивать колебания в механической системе на резонансной частоте с предельной амплитудой, что обеспечит повышение энергоэффективности и производительности вибрационного электромагнитного активатора.
\end{abstract}

Цель: провести аналитические исследования, позволяющие обеспечивать резонансные колебания с предельной амплитудой в вибрационном электромагнитном активаторе.

Методы исследования основаны на использовании обыкновенных дифференциальных уравнений, преобразования Лапласа, амплитудно-частотных характеристик, систем нелинейных алгебраических уравнений, спектрального анализа, сопоставления аналитических и экспериментальных характеристик.

Результаты. На основе линеаризованной математической модели механической системы вибрационного электромагнитного активатора рассмотрен режим свободных колебаний якоря-активатора с предельной амплитудой. Режим вынужденных колебаний предлагается рассматривать как естественное дополнение к режиму свободных колебаний с вынуждающей силой, действующей половину периода и имеющей специальную форму. Показано, что режимы свободных и вынужденных колебаний существенно зависят от параметров механической системы, которые определяются свойствами обрабатываемой жидкой среды. Проведен спектральный анализ вынуждающей силы, обеспечивающей резонансные колебания с предельной амплитудой в механической системе вибрационного электромагнитного активатора. Предложено техническое решение, обеспечивающее управление вибрационным электромагнитным активатором с автоматической настройкой на резонансную частоту и предельную амплитуду колебаний якоря-активатора. Такой режим обеспечивает максимизацию энергоэффективности и производительности процессов перемешивания жидких обрабатываемых сред. На основе сопоставления аналитических и экспериментальных частотных характеристик подтверждена гипотеза о допустимости линеаризации математической модели механической системы вибрационного электромагнитного активатора при аналитическом исследовании вынуждающей силы оптимальной формы.

\section{Ключевые слова:}

Буровой раствор, высоковязкий нефтепродукт, вибрационный электромагнитный активатор, резонанс, вынуждающая колебания сила, свободные колебания, вынужденные колебания, спектральный анализ, настройка на резонансную частоту, оценка погрешности.

\section{Введение}

В качестве источников возбуждения колебаний механических систем применяются вибровозбудители различных типов: гидравлические, пневматиче- ские и электромагнитные. При этом решаются задачи концентрации энергии колебаний в узкой частотной области и активного демпфирования колебаний в заданных областях частотного спектра $[1,2]$. 
Электромагнитные вибраторы (ЭМВ) как электрические машины возвратно-поступательного движения находят применение в технологиях, связанных с поиском, добычей и переработкой георесурсов.

В ряде конструкций ЭМВ способны эффективно генерировать низкочастотные колебания, являющиеся зондирующими воздействиями на грунт при сейсморазведке [3-8].

При перемешивании и разжижении высоковязких нефтепродуктов наряду с винтовыми устройствами, размывающими донные отложения с помощью турбулентных струй [9-15], эффективно используются вибрационные электромагнитные активаторы (ВЭМА), которые представляют из себя ЭМВ, создающие затопленные импульсные турбулентные струи за счёт специальной конструкции якоря-активатора [16-18].

Одним из существенных результатов применения ВЭМА на этапе подготовки работ по бурению скважин [19] стало то, что время приготовления и коррекции бурового раствора при использовании оборудования виброструйной магнитной активации в несколько раз меньше по сравнению с традиционными механическими центробежными мешалками [20].

Прогнозирование работоспособности и расчёт показателей качества ЭМВ, ВЭМА, линейных электрических машин возвратно-поступательного движения традиционно проводится на основе исследования динамики механических систем, работающих в колебательном режиме, при этом обоснованно применяются как линейные, так и нелинейные математические модели и схемы замещения [21-23].

\section{Резонансные колебания с предельной амплитудой в механической системе ВЭМА}

Рассмотрим на основе линейной математической модели процесс формирования резонансных колебаний с предельной амплитудой в механической системе ВЭМА при вынуждающей силе, действующей строго на половине периода резонансных колебаний. Погрешность применения именно линейной математической модели будет оценена в последнем разделе данной статьи.

Механический контур ВЭМА работает на резонансной частоте, и колебание якоря-активатора имеет полупериод свободных колебаний и полупериод вынужденных колебаний. Полупериод свободных колебаний начинается, когда притянутый к стенке якорь-активатор отталкивается под действием запасённой в пружине потенциальной энергии, при этом скорость сначала возрастает до максимума, затем спадает до нуля - якорь достигает максимального расстояния от стенки, а зазор в магнитной цепи также достигает максимума. C этого момента наступает режим вынужденных колебаний. В этом режиме якорь-активатор по гармоническому закону под действием вынуждающей стягивающей зазор электромагнитной силы притягивается точно к стенке, причём за то же время, за которое он от неё оттолкнулся. На этом основывается утверждение, что механический контур работает на резонансной частоте. Вынуждающая сила в режиме вынужденных колебаний имеет такую величину и форму, что якорь-активатор двигается по гармоническому закону.

Анализ свободно-вынужденных колебаний показывает принципиальную возможность построения нагруженных колебательных механических систем с максимальным КПД и производительностью и дает рекомендации по их построению.

Можно предположить, что результаты анализа механических систем с максимальным КПД и производительностью применимы и для других подобных колебательных систем, например электрических и гидравлических.

Анализ свободно-вынужденных колебаний в механической системе ВЭМА показывает, что потребляемая механическая мощность на резонансной частоте имеет предельный минимум, прямо пропорционально зависящий только от величины механического сопротивления. Работа ВЭМА в режиме свободно-вынужденных колебаний позволяет получить предельную экономию потребляемой электрической энергии, что очень важно с практической точки зрения.

Для получения максимальной производительности ВЭМА зазор в магнитной цепи при колебаниях якоря-активатора должен выбираться полностью. Для получения максимального КПД колебания якоря-активатора ВЭМА должны происходить по гармоническому закону с частотой, равной резонансной частоте.

Уравнение динамического равновесия механического контура, в соответствии с принципом Д'Аламбера, имеет вид:

$$
F_{\text {эм }}(t)=m_{\Sigma} \frac{d^{2}}{d t^{2}} x(t)+R_{\text {мех }} \frac{d}{d t} x(t)+x(t) k_{\text {пр }},
$$

где $F_{\text {эм }}(t)$ - сила, стягивающая магнитный зазор, являющаяся вынуждающей; $m_{\Sigma}=1,157$ кг - колеблющаяся масса; $R_{\text {мех }}=100 \mathrm{H} \cdot \mathrm{c} / \mathrm{m}-$ коэффициент вязкого трения (механическое сопротивление); $k_{\text {пр }}=1,85 \cdot 10^{5} \mathrm{H} / \mathrm{м}$ - жёсткость пружины; $x(t)$ - отклонение активатора от положения статического равновесия.

Применив преобразование Лапласа, получим:

$$
F_{\text {эм }}(p)=m_{\Sigma} x(p)\left(p^{2}+\frac{R_{\mathrm{Mex}}}{m_{\Sigma}} p+\frac{k_{\text {пр }}}{m_{\Sigma}}\right) .
$$

Выразим из (2) характеристическое уравнение $p^{2}+\frac{R_{\text {мех }}}{m_{\Sigma}} p+\frac{k_{\text {пр }}}{m_{\Sigma}}=0$ при выполнении условия $\left(\frac{R_{\text {мех }}}{m_{\Sigma}}\right)^{2}<\frac{k_{\text {пр }}}{m_{\Sigma}}$ имеет два комплексно-сопряжённых корня 


$$
\begin{aligned}
& p_{1}=-\left(\frac{R_{\text {mex }}}{2 m_{\Sigma}}\right)+\sqrt{\frac{k_{\text {mp }}}{m_{\Sigma}}-\left(\frac{R_{\text {mex }}}{2 m_{\Sigma}}\right)^{2}}=-\lambda+j \omega_{0}, \\
& p_{2}=-\left(\frac{R_{\text {mex }}}{2 m_{\Sigma}}\right)-\sqrt{\frac{k_{\text {mp }}}{m_{\Sigma}}-\left(\frac{R_{\text {mex }}}{2 m_{\Sigma}}\right)^{2}}=-\lambda-j \omega_{0} .
\end{aligned}
$$

Решив уравнение $\left(\frac{R_{\text {мех }}}{m_{\Sigma}}\right)^{2}-\frac{k_{\text {пр }}}{m_{\Sigma}}=0$, получим величину граничного коэффициента вязкого трения $R_{\text {меххгг }}=2 \sqrt{k_{\text {пр }} m_{\Sigma}}=925,3 \mathrm{H} \cdot \mathrm{c} / \mathrm{M}$. баний

Круговая и линейная частота свободных коле-

$$
\omega_{0}=\sqrt{\frac{k_{\mathrm{mp}}}{m_{\Sigma}}-\left(\frac{R_{\mathrm{mex}}}{2 m_{\Sigma}}\right)^{2}}=2 \pi \cdot 63,2=397 \mathrm{paz} / \mathrm{c}
$$

или $f_{0}=63,2$ Гц.

Исследуем, как зависит $\omega_{0}$ от $R_{\text {мех }}$ и $m_{\Sigma}$ (рис. 1 ).
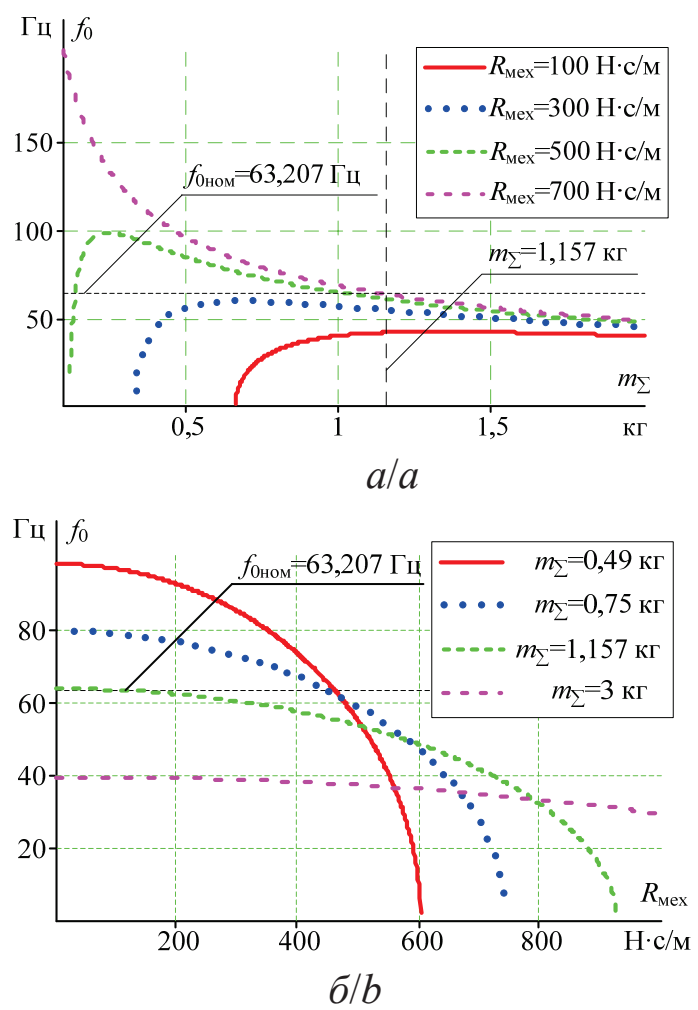

Pис.1. Характеристики частоты свободных колебаний меха нической систелы от: а) сумларной массы якоря-активатора; б) коэффиииента демпфирования

Fig. 1. Dependence of the frequency of mechanical system free oscillations on: a) total mass of the armature-activator; $b$ ) dam ping coefficient

Величина приращения резонансной частоты (табл. 1) при постоянной жёсткости пружины и при изменении вязкого трения определяется как

$$
\Delta f_{\mathrm{pe} 3} \%=\Delta \omega_{R \mathrm{M}} \%=\frac{\omega_{R \mathrm{M} 1}-\omega_{R \mathrm{M}}}{\omega_{R \mathrm{M} 1}} \cdot 100 \%,
$$

где $R_{\mathrm{m} 1}=100 \mathrm{H} \cdot \mathrm{c} / \mathrm{m}$ - начальное значение коэффициента вязкого трения.
Таблица 1. Приращениерезонансной частоты при $k_{\mathrm{mp}}=1,85 \cdot 10^{5} \mathrm{H} / \mathrm{m}$ $u R_{\text {мех }}=v a r$

Table 1. Increment of the resonant frequency at $k_{\mathrm{mp}}=1,85 \cdot 10^{5} \mathrm{~N} / \mathrm{m}$ and $R_{\text {wex }}=$ var

\begin{tabular}{|c|c|c|c|c|}
\hline$R_{\text {nex }}, \mathrm{H} \cdot \mathrm{c} / \mathrm{M} / \mathrm{N} \cdot \mathrm{s} / \mathrm{m}$ & 100 & 300 & 500 & 700 \\
\hline$f_{\text {pes }}, \mathrm{\Gamma} \amalg / \mathrm{Hz}$ & 63,27 & 60,20 & 53,55 & 34,22 \\
\hline$\Delta f_{\text {pes }}, \%$ & 0 & 4,84 & 15,36 & 41,62 \\
\hline
\end{tabular}

Согласно табл. 1 , при увеличении $R_{\text {мех }}$ в 7 раз $\Delta f_{\text {рез }} \%=41,62 \%$, т. е. изменяется незначительно.

Логарифмический декремент затухания

$$
\lambda=\frac{R_{\text {mex }}}{2 m_{\Sigma}}=43,215 \mathrm{c}^{-1} .
$$

Показатель колебательности механического контура

$$
\chi=\frac{\omega_{0}}{\lambda}=\sqrt{\frac{4 k_{\mathrm{mp}} m_{\Sigma}}{R_{\mathrm{mex}}^{2}}-1}=9,199 .
$$

Добротность механического контура

$$
Q=\frac{\sqrt{m_{\Sigma} k_{\text {пр }}}}{R_{\text {мех }}}=4,626
$$

Так как $Q=4,626<10$, следовательно, механический контур является нагруженным.

Механический контур ВЭМА на резонансной частоте может работать в режимах вынужденных и свободных колебаний. В режиме вынужденных колебаний якорь-активатор притягивается к стенке корпуса под действием электромагнитной вынуждающей силы, а в режиме свободных колебаний якорь отталкивается. Притянем якорь-активатор к стенке корпуса, потом отпустим. В этом случае активатор будет совершать затухающие колебания, которые описываются зависимостью

$$
\delta_{\text {св }}(t)=\delta_{0}-\left(\delta_{0}-\delta_{\text {ст }}\right) \cos \left(\omega_{0} t\right) e^{-\lambda t} .
$$

При $R_{\text {мех }}=100 \mathrm{H} \cdot \mathrm{c} /$ м за половину периода свободные колебания затухают в 1,414 раза, за один период в 2 раза, а за 6 периодов в 60 раз (рис. 2).

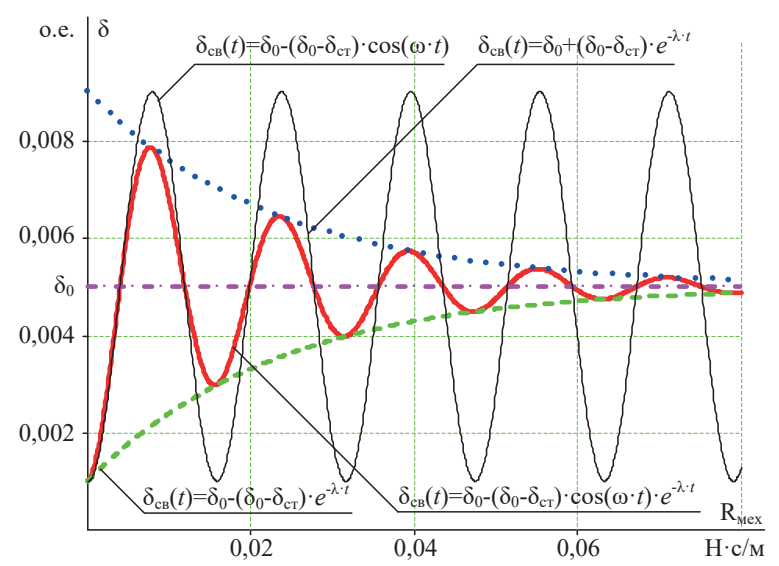

Pис. 2. Переходный процесс свободных колебаний якоря-активаmopa

Fig. 2. Free oscillations response of armature-activator 
Процесс затухающих колебаний при разных коэффициентах вязкого трения $\left(R_{\text {wех }}\right)$ представлен на рис. 3.

За половину периода якорь-активатор оттолкнётся от стенки на расстояние

$$
\delta_{\mathrm{M}}=\delta_{0}-\left(\delta_{0}-\delta_{\text {ст }}\right) \cos (\pi) e^{-\lambda \frac{\pi}{\omega_{0}}}
$$

- максимальный зазор в магнитной цепи в колебательном процессе. Это расстояние можно для удобства выразить в процентах $\delta_{\text {м }} \%=\frac{\delta_{\text {м }}}{\left(2 \delta_{0}-\delta_{\text {ст }}\right)} \cdot 100 \%$, и оно тем меньше, чем больше коэффициент вязкого трения.

При стремлении коэффициента вязкого трения к граничному значению $R_{\text {мех гг }}=2 \sqrt{k_{\text {пр }} m_{\Sigma}}=925,3 \mathrm{H} \cdot \mathrm{c} / \mathrm{M}$ якорь-активатор отталкивается от стенки и стремится принять положение статического равновесия $\delta_{\mathrm{x}}=\delta_{0}=0,005 \mathrm{м}$, характер же переходного процесса при этом становится неколебательным.

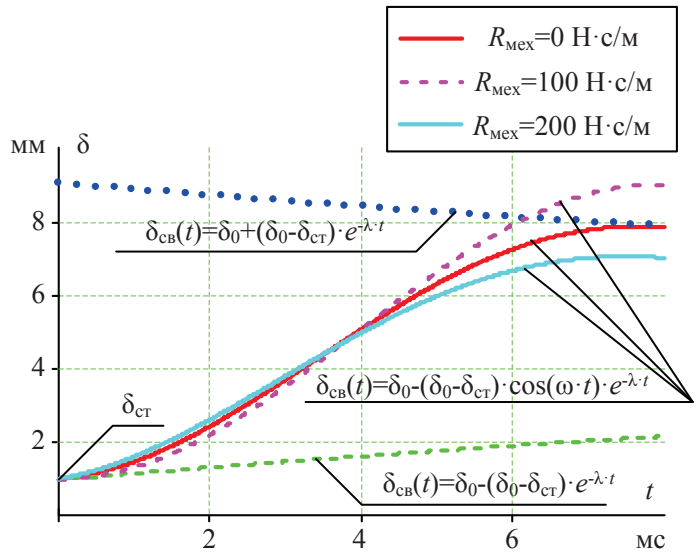

Pис. 3. Переходный процесс отскока от стенки корпуса ВЭМА притянутого якоря-активатора на полупериоде свобод ных колебаний при варьировании коэффициента демпфирования

Fig. 3. Transitional characteristic of the attracted anchor-activator rebounding from the housing side of the vibration electromag netic activators on the half-period of free oscillations by varying the damping coefficient

Проанализировав зависимости (рис. 3), можно видеть, что рабочая точка хорошо сбалансирована, т. е. $\omega_{0}, Q, \delta_{\mathrm{m}}, \delta_{\mathrm{m}} \%$ находятся в области незначительного приращения $\Delta \omega_{0}, \Delta Q, \Delta \delta_{w}, \delta_{v} \%$ при варьировании параметров механического контура $R_{\text {мех }}, m_{\Sigma}$

В первом полупериоде якорь-активатор под действием силы пружины отталкивается от стенки и совершает свободные колебания на собственной частоте $\omega_{0}$, зазор магнитной цепи при этом достигает максимального значения $\delta_{x}$. Во втором полупериоде колебания происходят под действием электромагнитной вынуждающей силы, в результате чего якорь снова притянется к стенке.

Пропорционально отклонению активатора от положения равновесия в режиме свободных колебаний

$$
x(t)=\delta_{0}-\delta_{\text {св }}(t)=\left(\delta_{0}-\delta_{\text {ст }}\right) \cos \left(\omega_{0} t\right) e^{-\lambda t}
$$

изменяется сила пружины

$$
F_{\text {пр_св }}(t)=k_{\text {пр }}\left(\delta_{0}-\delta_{\text {св }}(t)\right)=k_{\text {пр }}\left(\delta_{0}-\delta_{\text {ст }}\right) \cos \left(\omega_{0} t\right) e^{-\lambda t} \text {. }
$$

Возьмём производную с учётом выражения (3) и определим скорость в режиме свободных колебаний

$$
\begin{aligned}
& v_{\mathrm{cв}}(t)=\frac{d\left(\delta_{0}-\delta_{\text {св }}(t)\right)}{d t}=\frac{d\left(\left(\delta_{0}-\delta_{\text {ст }}\right) \cos \left(\omega_{0} t\right) e^{-\lambda t}\right)}{d t} \\
& v_{\text {св }}(t)=\frac{-1}{2}\left(\delta_{0}-\delta_{\text {ст }}\right) \sin \left[\frac{1}{2}\left(4 \frac{k_{\text {пр }}}{m_{\Sigma}}-\frac{R_{\text {мех }}^{2}}{m_{\Sigma}^{2}}\right)^{\frac{1}{2}} t\right] \times \\
& \times\left(4 \frac{k_{\text {пр }}}{m_{\Sigma}}-\frac{R_{\text {мex }}^{2}}{m_{\Sigma}^{2}}\right)^{\frac{1}{2}} \exp \left(-\frac{1}{2} \frac{R_{\text {мex }}}{m_{\Sigma}} t\right)-\frac{1}{2}\left(\delta_{0}-\delta_{\text {ст }}\right) \times \\
& \times \cos \left[\frac{1}{2}\left(4 \frac{k_{\text {пр }}}{m_{\Sigma}}-\frac{R_{\mathrm{mex}}^{2}}{m_{\Sigma}^{2}}\right)^{\frac{1}{2}} t\right] \frac{R_{\mathrm{mex}}}{m_{\Sigma}} \exp \left(-\frac{1}{2} \frac{R_{\mathrm{mex}}}{m_{\Sigma}} t\right),
\end{aligned}
$$

пропорционально которой изменяется диссипативная сила $F_{\text {диссев }}(t)=R_{\text {мех }} v_{\text {св }}(t)$.

Возьмём вторую производную, с учётом выражения (3) определим ускорение в режиме свободных колебаний

$$
\begin{aligned}
& a_{\mathrm{cB}}(t)=\frac{d^{2}\left(\delta_{0}-\delta_{\mathrm{cB}}(t)\right)}{d t^{2}}= \\
& =\frac{d^{2}\left(\left(\delta_{0}-\delta_{\text {ст }}\right) \cos \left(\omega_{0} t\right) e^{-\lambda t}\right)}{d t^{2}}, \\
& a_{\text {св }}(t)=\frac{1}{4}\left(-\delta_{0}-\delta_{\text {ст }}\right) \cos \left[\frac{1}{2}\left(4 \frac{k_{\text {пр }}}{m_{\Sigma}}-\frac{R_{\text {мех }}^{2}}{m_{\Sigma}^{2}}\right)^{\frac{1}{2}} t\right] \times \\
& \times\left(4 \frac{k_{\text {пр }}}{m_{\Sigma}}-\frac{R_{\text {мex }}^{2}}{m_{\Sigma}^{2}}\right)^{\frac{1}{2}} \exp \left(-\frac{1}{2} \frac{R_{\text {мex }}}{m_{\Sigma}} t\right)-\frac{1}{4}\left(\delta_{0}-\delta_{\text {ст }}\right) \times \\
& \times \sin \left[\frac{1}{2}\left(4 \frac{k_{\text {пр }}}{m_{\Sigma}}-\frac{R_{\text {mex }}^{2}}{m_{\Sigma}^{2}}\right)^{\frac{1}{2}} t\right]\left(4 \frac{k_{\text {пр }}}{m_{\Sigma}}-\frac{R_{\text {mex }}^{2}}{m_{\Sigma}^{2}}\right)^{\frac{1}{2}} \frac{R_{\text {mex }}}{m_{\Sigma}} \times \\
& \times \exp \left(-\frac{1}{2} \frac{R_{\text {мех }}}{m_{\Sigma}} t\right)+\frac{1}{4}\left(\delta_{0}-\delta_{\text {ст }}\right) \times \\
& \times \sin \left[\frac{1}{2}\left(4 \frac{k_{\text {пр }}}{m_{\Sigma}}-\frac{R_{\text {мex }}^{2}}{m_{\Sigma}^{2}}\right)^{\frac{1}{2}} t\right]\left(4 \frac{k_{\text {пр }}}{m_{\Sigma}}-\frac{R_{\text {мeх }}^{2}}{m_{\Sigma}^{2}}\right)^{\frac{1}{2}} \frac{R_{\text {мex }}}{m_{\Sigma}} \times \\
& \times \exp \left(-\frac{1}{2} \frac{R_{\mathrm{mex}}}{m_{\Sigma}} t\right)+\frac{1}{4}\left(\delta_{0}-\delta_{\text {ст }}\right) \times \\
& \times \cos \left[\frac{1}{2}\left(4 \frac{k_{\text {пр }}}{m_{\Sigma}}-\frac{R_{\mathrm{mex}}^{2}}{m_{\Sigma}^{2}}\right)^{\frac{1}{2}} t\right] \frac{R_{\mathrm{mex}}^{2}}{m_{\Sigma}^{2}} \exp \left(-\frac{1}{2} \frac{R_{\mathrm{mex}}}{m_{\Sigma}} t\right) .
\end{aligned}
$$




$$
\begin{gathered}
=\frac{1}{4} \cos \left[\frac{1}{2}\left(4 \frac{k_{\mathrm{mp}}}{m_{\Sigma}}-\frac{R_{\mathrm{cm}}^{2}(t)=}{m_{\Sigma}^{2}}\right)^{\frac{1}{2}} t\right] \exp \left(-\frac{1}{2} \frac{R_{\mathrm{mex}}}{m_{\Sigma}} t\right) \times \\
\times \frac{-4 \delta_{0} k_{\mathrm{mp}} m_{\Sigma}+\delta_{0} R_{\mathrm{mex}}^{2}+4 \delta_{\mathrm{cr}} k_{\mathrm{mp}} m_{\Sigma}-\delta_{\mathrm{cr}} R_{\mathrm{mex}}^{2}}{m_{\Sigma}^{2}}- \\
-\frac{1}{2}\left(-\delta_{0}+\delta_{\mathrm{cr}}\right) \sin \left[\frac{1}{2}\left(4 \frac{k_{\mathrm{mp}}}{m_{\Sigma}}-\frac{R_{\mathrm{mex}}^{2}}{m_{\Sigma}^{2}}\right)^{\frac{1}{2}} t\right] \times \\
\times\left(\frac{4 k_{\mathrm{mp}} m_{\Sigma}-R_{\mathrm{mex}}^{2}}{m_{\Sigma}^{2}}\right)^{\frac{1}{2}} \frac{R_{\mathrm{mex}}}{m_{\Sigma}} \exp \left(-\frac{1}{2} \frac{R_{\mathrm{mex}}}{m_{\Sigma}} t\right)- \\
-\frac{1}{4}\left(-\delta_{0}+\delta_{\mathrm{cr}}\right) \cos \left[\frac{1}{2}\left(\frac{4 k_{\mathrm{mp}} m_{\Sigma}-R_{\mathrm{mex}}^{2}}{m_{\Sigma}^{2}}\right)^{\frac{1}{2}} t\right] \times \\
\times \frac{R_{\mathrm{mex}}^{2}}{m_{\Sigma}^{2}} \exp \left(-\frac{1}{2} \frac{R_{\mathrm{mex}}}{m_{\Sigma}} t\right) .
\end{gathered}
$$

Используя выражение (5), запишем выражение для силы инерции якоря-активатора в режиме свободных колебаний

$$
F_{\text {ин_св }}(t)=m_{\Sigma} a_{\text {св }}(t) .
$$

Предельная диссипативная мощность механического контура ВЭМА, работающего на резонансной частоте, прямо пропорциональна величине коэффициента вязкого трения и обратно пропорциональна толщине стенки корпуса. При номинальных параметрах механического контура ВЭМА в гидравлический контур может быть передано не более 345,18 Вт, подразумевается также, что ВЭМА работает в безударном режиме.

При стремлении коэффициента вязкого трения к бесконечно большой величине $\delta_{\text {м }}$ стремится к значению, равному величине зазора в положении статического равновесия. За половину периода якорь-активатор оттолкнётся от стенки на расстояние $\delta_{\mathrm{m}}$. Чтобы колебания были незатухающие во втором полупериоде нужно к якорю приложить силу такой формы и величины, чтобы он снова полностью притянулся к стенке, при этом колебание якоря можно записать как:

$$
\begin{gathered}
\delta_{\text {вын }}(t)= \\
=\frac{\delta_{\mathrm{M}}-\delta_{\text {ст }}}{2}\left(1+\cos \left(\sqrt{\frac{k_{\text {пр }}}{m_{\Sigma}}-\left(\frac{R_{\text {мех }}}{m_{\Sigma}}\right)^{2}} t\right)\right)+\delta_{\text {ст }} .
\end{gathered}
$$

Установившиеся колебания в механической системе ВЭМА, работающей на резонансной частоте, являются свободно-вынужденными и описываются согласно (3) в режиме свободных колебаний, а согласно (6) в режиме вынужденных колебаний с частотой вынуждающей силы, равной резонансной частоте механического контура.

Представляется важным в режиме вынужденных колебаний в механической системе получить и проанализировать аналитическое выражение для электролагнитной вынуждающей колебания силы, которая якорь-активатор, оттолкнувшийся от стенки на максимальное расстояние, вновь притянет к стенке. Для получения наибольшего КПД и производительности якорь-активатор должен колебаться с частотой, равной частоте собственных колебаний $\omega_{0}=2 \pi f_{0}$, и с амплитудой, равной наибольшей величине зазора магнитной цепи $\delta_{0}-\delta_{\text {сг }}$. Большую производительность, чем предельная, можно получить только при увеличении зазора магнитной цепи в положении статического равновесия. Если же зазор в положении равновесия оставить прежним, то максимальный КПД и производительность можно получить, заставив якорь колебаться согласно выражениям (3) и (6). Электромагнитная вынуждающая сила должна быть отлична от нуля только во втором полупериоде колебаний, когда якорь притягивается к стенке. Когда же якорь отталкивается от стенки под действием силы пружины и внешней вынуждающей силы нет, то колебание происходит в свободном режиме с собственной частотой. В режиме вынужденных колебаний механический контур ВЭМА получает энергию, которую он израсходовал в течение предыдущего полупериода свободных колебаний (рис. 4). Используя первую и вторую производные виброперемещения (3), а также уравнение динамического равновесия для механического контура (1), можно получить искомое аналитическое выражение для электромагнитной вынуждающей колебания силы.

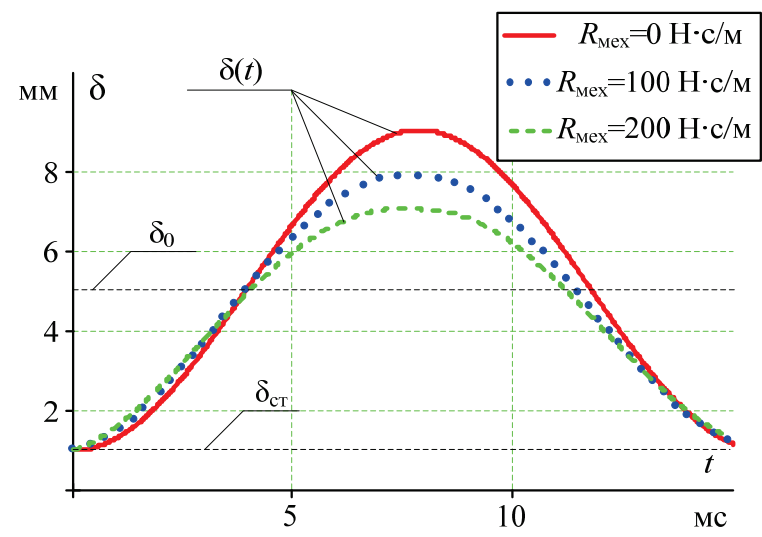

Pис.4. Свободно-вынужденные колебания якоря-активатора ВЭМА при варьировании коэффициента вязкого трения

Fig. 4. Free-forced oscillations of the armature-activator vibration electromagnetic activators with varying viscous friction

Выражение для отклонения якоря-активатора от положения статического равновесия с учётом (6) 


$$
\begin{gathered}
x_{\text {вын }}(t)=-1\left(\frac{\delta_{\mathrm{M}}-\delta_{\text {ст }}}{2}(1+\cos (\omega t))+\delta_{\text {ст }}-\delta_{0}\right), \\
x_{\mathrm{B̆H}}(t)=\delta_{0}-\delta_{\text {ст }}-\left(1+\cos \left(\sqrt{\frac{k_{\text {пр }}}{m_{\Sigma}}-\left(\frac{R_{\mathrm{mex}}}{2 m_{\Sigma}}\right)^{2}} t\right)\right) \times \\
\times \frac{1}{2}\left(\delta_{0}-\left(\delta_{0}-\delta_{\text {ст }}\right) \exp \left(-\frac{R_{\mathrm{mex}}}{2 m_{\Sigma}} \frac{\pi}{\sqrt{\frac{k_{\text {пр }}}{m_{\Sigma}}-\left(\frac{R_{\text {мех }}}{2 m_{\Sigma}}\right)^{2}}}\right) .\right.
\end{gathered}
$$

Первая производная - скорость в режиме вынужденных колебаний

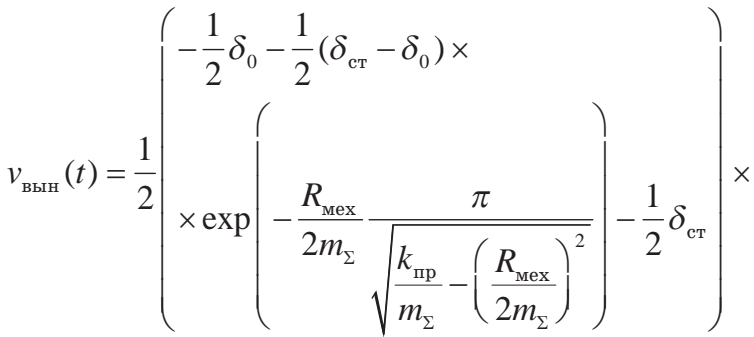

$$
\begin{aligned}
& \times \sin \left[\frac{1}{2}\left(4 \frac{k_{\text {mp }}}{m_{\Sigma}}-\frac{R_{\text {mex }}^{2}}{m_{\Sigma}^{2}}\right)^{\frac{1}{2}} t\right] \sqrt{4 \frac{k_{\text {mp }}}{m_{\Sigma}}-\frac{R_{\text {mex }}^{2}}{m_{\Sigma}^{2}}} .
\end{aligned}
$$

Вторая производная - ускорение в режиме вынужденных колебаний

$$
\begin{aligned}
& a_{\text {вын }}(t)=\frac{1}{2}\left(\begin{array}{l}
\frac{1}{4} \delta_{0}-\frac{1}{4}\left(\delta_{\text {ст }}-\delta_{0}\right) \times \\
\left.\times \exp \left(\frac{-R_{\text {мех }}}{m_{\Sigma}} \frac{\pi}{\sqrt{4 \frac{k_{\text {пр }}}{m_{\Sigma}}-\frac{R_{\text {мех }}^{2}}{m_{\Sigma}^{2}}}}\right)-\frac{1}{4} \delta_{\text {ст }}\right) \times
\end{array}\right. \\
& \times \cos \left[\frac{1}{2}\left(4 \frac{k_{\text {mp }}}{m_{\Sigma}}-\frac{R_{\text {sex }}^{2}}{m_{\Sigma}^{2}}\right)^{\frac{1}{2}} t\right] \sqrt{4 \frac{k_{\text {mp }}}{m_{\Sigma}}-\frac{R_{\text {мex }}^{2}}{m_{\Sigma}^{2}}} .
\end{aligned}
$$

Желаемая электромагнитная вынуждающая колебания сила в режиме вынужденных колебаний

$$
F_{\text {жел }}(t)=m_{\Sigma} \frac{d^{2}}{d t^{2}} x_{\text {вын }}(t)+R_{\text {мех }} \frac{d}{d t} x_{\text {вын }}(t)+x_{\text {вын }}(t) k_{\text {пр }} \text {. }
$$

Необходимая величина амплитуды желаемой электромагнитной вынуждающей силы в режиме вынужденных колебаний зависит от $m_{\Sigma}, R_{\text {мех }}$ и $\delta_{\text {ст }}$

$$
\begin{aligned}
& F_{\text {жел_макс }}\left(R_{\text {мех }}, m_{\Sigma}, \delta_{\text {ст }}\right)= \\
& R_{\text {мех }}\left(\begin{array}{l}
\frac{1}{4} \delta_{0}-\frac{1}{4}\left(-\delta_{0}+\delta_{\text {ст }}\right) \times \\
\times \exp \left(\frac{-R_{\text {мex }}}{m_{\Sigma}} \frac{\pi}{\sqrt{4 \frac{k_{\text {пр }}}{m_{\Sigma}}-\frac{R_{\text {Mex }}^{2}}{m_{\Sigma}^{2}}}}\right)-\frac{1}{4} \delta_{\text {ст }}
\end{array}\right) \times
\end{aligned}
$$

$$
\begin{aligned}
& \times \sqrt{4 \frac{k_{\text {пр }}}{m_{\Sigma}}-\frac{R_{\text {мex }}^{2}}{m_{\Sigma}^{2}}}+ \\
& +k_{\text {пр }}\left(\begin{array}{l}
\frac{1}{2} \delta_{0}+\frac{1}{2}\left(-\delta_{0}+\delta_{\text {ст }}\right) \times \\
\times \exp \left(\frac{-R_{\text {мех }}}{m_{\Sigma}} \frac{\pi}{\sqrt{4 \frac{k_{\text {пр }}}{m_{\Sigma}}-\frac{R_{\text {мех }}^{2}}{m_{\Sigma}^{2}}}}\right)-\frac{1}{2} \delta_{\text {ст }}
\end{array}\right) .
\end{aligned}
$$

Необходимая величина амплитуды электромагнитной вынуждающей силы в режиме вынужденных колебаний обратно пропорциональна добротности контура $Q$ и прямо пропорциональна толщине стенки $\delta_{\text {ст. }}$.

Выражение для электромагнитной вынуждающей колебания силы оптимальной формы в течение половины периода вынужденных колебаний имеет вид

$$
F_{\text {вын }}(t)= \begin{cases}F_{\text {жел }}(t) \text { если } \sin \left(\omega_{0} t\right) \geq 0 \\ 0 \quad \text { иначе }\end{cases}
$$

а в течение полупериода свободных колебаний вынуждающая сила равна нулю (рис. 5).

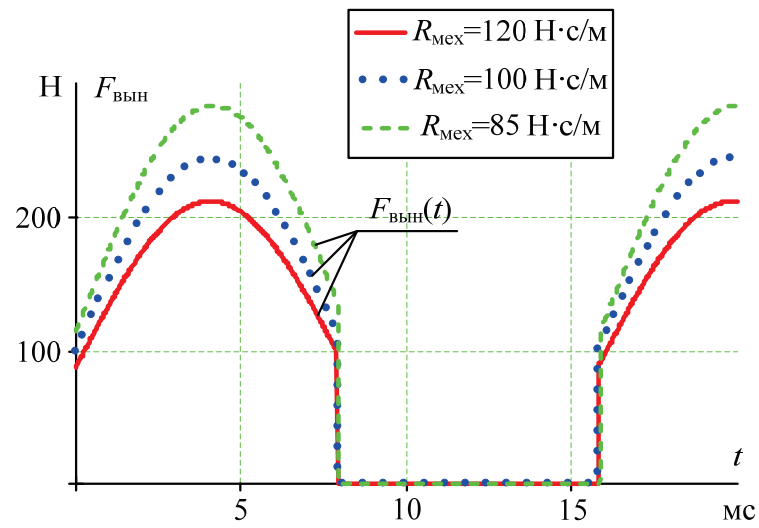

Pис. 5. Вид вынуждающей силы в течение периода, обеспечивающей резонансные колебания с предельной амплитудой в механической системе ВЭМА при варьировании коэффиииента вязкого трения

Fig. 5. Type of driving force during the period providing resonant oscillations with a limiting amplitude in the vibration electromagnetic activator's mechanical system with varying viscous friction coefficient

Следующей задачей является спектральный анализ этой силы. Разложим электромагнитную вынуждающую колебания силу оптимальной формы до десятой гармоники и получим её ортогональный (синусно-косинусный - табл. 2) и амплитудно-фазовый спектры (табл. 3).

Постоянная составляющая:

$$
F_{0}(t)=\frac{\omega_{0}}{\pi} \int_{\frac{\pi}{2 \omega_{0}}}^{\frac{5 \pi}{2 \omega_{0}}} F_{\text {вын }}(t) d t .
$$

Восстановленный с точностью до десятой гармоники сигнал силы имеет вид: 


$$
\begin{gathered}
F F(t)=F_{0}+\sum_{k=1}^{k=10}\left(F_{k} \cos (k \omega t)+F_{k 1} \sin (k \omega t)\right), \\
A F_{k}=\sqrt{F_{k}^{2}+F_{k 1}^{2}}, \alpha F_{k}=\operatorname{arctg}\left(\frac{F_{k}}{F_{k 1}}\right) .
\end{gathered}
$$

таблица 2. Результаты ортогонального спектрального анализа Table 2. Results of orthogonal spectral analysis

\begin{tabular}{|c|c|c|c|c|c|c|}
\hline \multicolumn{7}{|c|}{$R_{\text {мех }}=100 \mathrm{H} \cdot \mathrm{c} / \mathrm{m}(\mathrm{N} \cdot \mathrm{s} / \mathrm{m}), m_{\Sigma}=1,157$ кг $(\mathrm{kg}), k_{\text {пр }}=1,85 \cdot 10^{5} \mathrm{H} / \mathrm{m}(\mathrm{N} / \mathrm{m})}$, \\
\hline$F_{0}$ & $F_{1}$ & $F_{11}$ & $F_{2}$ & $F_{21}$ & $F_{3}$ & $F_{31}$ \\
\hline 98,816 & $-3,7$ & 136,152 & $-28,9$ & $-3,14$ & 0 & 22,716 \\
\hline$F_{4}$ & $F_{41}$ & $F_{5}$ & $F_{51}$ & $F_{6}$ & $F_{61}$ & $F_{7}$ \\
\hline$-1,71$ & $-1,26$ & 0 & 13,629 & $-2,474$ & $-0,807$ & 0 \\
\hline$F_{71}$ & $F_{8}$ & $F_{81}$ & $F_{9}$ & $F_{91}$ & $F_{10}$ & $F_{101}$ \\
\hline 9,735 & $-1,374$ & $-0,598$ & 0 & 7,572 & $-0,875$ & $-0,475$ \\
\hline
\end{tabular}

Таблица 3. Результаты алплитудно-фазового анализа

Table 3. $\quad$ Results of magnitude-phase analysis

\begin{tabular}{|c|c|c|c|c|c|c|}
\hline \multicolumn{7}{|c|}{$R_{\text {мex }}=100 \mathrm{H} \cdot \mathrm{c} / \mathrm{M}(\mathrm{N} \cdot \mathrm{s} / \mathrm{m}), m_{\Sigma}=1,157 \mathrm{\kappa \Gamma}(\mathrm{kg}), k_{\mathrm{mp}}=1,85 \cdot 10^{5} \mathrm{H} / \mathrm{M}(\mathrm{N} / \mathrm{m})}$, \\
\hline$k$ & 0 & 1 & 2 & 3 & 4 & 5 \\
\hline$A F_{k}=5 \cdot 10^{-3} \mathrm{M}(\mathrm{m}), \delta_{\mathrm{cr}}=1 \cdot 10^{-3} \mathrm{M}(\mathrm{m})$ \\
\hline$\alpha F_{k}(360 / 2 \pi)$ & 96,816 & 136,202 & 32,937 & 22,716 & 5,907 & 13,629 \\
\hline$k$ & - & $-1,555$ & 83,796 & 0 & 77,734 & 0 \\
\hline$A F_{k}$ & 2,602 & 9,735 & 1,499 & 8,553 & 0,995 & \\
\hline$\alpha F_{k}(360 / 2 \pi)$ & 71,937 & $-22,1$ & 66,499 & 0 & 61,474 & \\
\hline
\end{tabular}

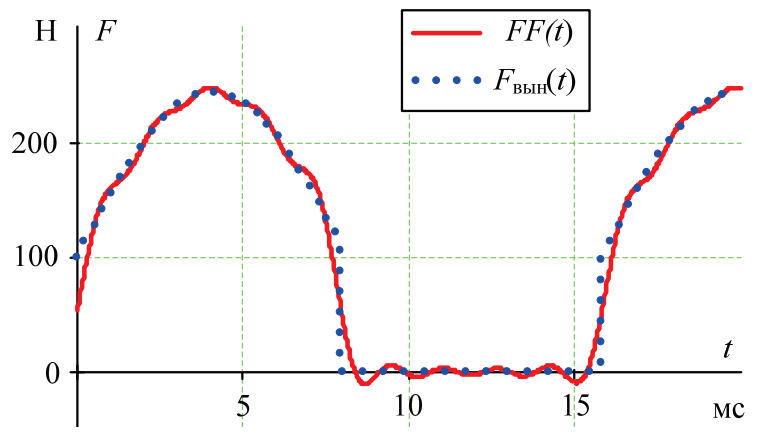

Pис. 6. Сравнение исходной и восстановленной после спектраль ного анализа по десяти гарлоникал вынуждающей силь

Fig. 6. Comparison of the original and reconstructed driving force after spectral analysis by ten harmonics

Оценим содержание первой гармоники с частотой, равной резонансной частоте механического контура, нулевой гармоники и высших гармоник (табл. 4).

В результате получено аналитическое описание идеальной электромагнитной вынуждающей колебания силы на резонансной частоте.

Теперь оценим степень подавления неосновных гармоник найденной оптимальной вынуждающей силы в резонансном режиме при заданной величине коэффициента вязкого трения. Для этого к уравнению (1) нужно применить преобразование Лапласа - получим операторное уравнение

$$
\begin{gathered}
F_{\text {эм }}(p)=m_{\Sigma} p^{2} x(p)+R_{\text {мех }} p x(p)+x(p) k_{\text {пр }}, \\
F_{\text {эм }}(p)=x(p)\left(m_{\Sigma} p^{2}+R_{\text {мех }} p+k_{\text {пр }}\right) .
\end{gathered}
$$

Таблица 4. Распределение гармонического состава вынуждающей колебания силы оптимальной формы

Table 4. Distribution of harmonic composition of optimal form driving force

\begin{tabular}{|c|c|c|}
\hline $\begin{array}{c}\text { Коэффициент основ- } \\
\text { ной гармоники силы } \\
\text { оптимальной формы } \\
\text { Coefficient of the main } \\
\text { harmonic of the opti- } \\
\text { mal form force }\end{array}$ & $\begin{array}{c}\text { Коэффициент нуле- } \\
\text { вой гармоники силы } \\
\text { oптимальной формы } \\
\text { Coefficient of the zero } \\
\text { harmonic of the opti- } \\
\text { mal form force }\end{array}$ & $\begin{array}{c}\text { Коэффициент вы- } \\
\text { сших гармоник силы } \\
\text { оптимальной формы } \\
\text { Coefficient of the } \\
\text { high-harmonics of the } \\
\text { optimal form force }\end{array}$ \\
\hline$k F_{1 \%}=\frac{A F_{1}}{\sum_{k=0}^{k=10} A F_{k}}$ & $k F_{0 \%}=\frac{A F_{0}}{\sum_{k=10}^{k=10} A F_{k}}$ & $k F_{\text {выс\% }}=\frac{\sum_{k=2}^{k=10} A F_{k}}{\sum_{k=0} A F_{k}}$ \\
\hline$k F_{1 \%}=41,689 \%$ & $k F_{0 \%}=29,634 \%$ & $k F_{\text {выс \% }}=28,677 \%$ \\
\hline
\end{tabular}

Зная, что $x(p)=\frac{v(p)}{p}$, получим передаточную функцию механического контура по скорости

$$
W_{V}(p)=\frac{v(p)}{F_{\text {эм }}(p)}, W_{V}(p)=\frac{p}{\left(m_{\Sigma} p^{2}+R_{\text {мех }} p+k_{\text {пр }}\right)} .
$$

Заменив $p$ на $j \omega$, получим комплексно-частотную характеристику механического контура по виброскорости

$$
\begin{gathered}
W_{V}(p)=P(\omega)+j Q(\omega)=A(\omega) e^{j \phi(\omega)}, \\
W_{V}(p)=\frac{j \omega}{\left(m_{\Sigma} j^{2} \omega^{2}+R_{\text {мех }} j \omega+k_{\text {пр }}\right)}= \\
=\frac{R_{\text {мех }} \omega^{2}+j \omega\left(k_{\text {пр }}-m_{\Sigma} \omega^{2}\right)}{\left(k_{\text {пр }}-m_{\Sigma} \omega^{2}\right)^{2}+R_{\text {мех }}^{2} \omega^{2}},
\end{gathered}
$$

где

$$
A(\omega)=\frac{\omega \sqrt{R_{\text {мех }}^{2}+\left(k_{\text {пр }}-m_{\Sigma} \omega^{2}\right)^{2}}}{\left(k_{\text {пр }}-m_{\Sigma} \omega^{2}\right)^{2}+R_{\text {мех }}^{2} \omega^{2}}
$$

- амплитудно-частотная характеристика (АЧХ) механического контура по виброскорости;

$$
\phi(\omega)=\operatorname{arctg}\left(\frac{k_{\text {пр }}-m_{\Sigma} \omega^{2}}{R_{\text {мех }} \omega}\right)
$$

- фазо-частотная характеристика (ФЧХ) механического контура по виброскорости.

Используя данные табл. 4, можно оценить степень подавления механическим контуром неосновных гармоник виброскорости для электромагнитной вынуждающей колебания силы оптимальной формы и для вынуждающей силы прямоугольной формы (табл. 5, 6).

Приращение суммарной колеблющейся массы вызывает уменьшение резонансной частоты, увеличивает добротность механического колебательного контура, а также уменьшает граничную величину коэффициентов вязкого трения и затухания, увеличивает показатель колебательности механического контура и максимальный размах колебаний на резонансной частоте. Кроме того, увеличение суммарных колеблющихся масс (за счёт присоединённых масс обрабатываемой жидкости) при- 
водит к снижению предельного виброускорения в режиме свободно-вынужденных колебаний. $\mathrm{K}$ сожалению, из-за насыщения магнитной цепи нет возможности получить вынуждающую электромагнитную силу большую, чем это позволяет магнитный поток насыщения, который обусловлен магнитными свойствами стали магнитопровода, следовательно, для получения предельного виброускорения при максимальной стягивающей магнитный зазор силе нужно либо снижать суммарную колеблющуюся массу, либо увеличивать жёсткость пружины.

Таблица 5. Оценка подавления механическим контуром ВЭМА неосновных гармоник виброскорости при разных величинах коэффиииента вязкого трения $\left(R_{\text {sex__p }_{\text {_p }}}=2 \sqrt{k_{\text {пр }} m_{\Sigma}}=925,3 H \cdot c / \mathrm{m}\right)$

Table 5. Estimation of suppression of minor harmonics by the vibration electromagnetic activator mechanical contour of vibration velocity with varying values of mechanical resistance $\left(R_{\text {Mex } \_ \text {гp }}=2 \sqrt{k_{\text {пp }} m_{\Sigma}}=925,3 \mathrm{~N} \cdot \mathrm{s} / \mathrm{m}\right)$

\begin{tabular}{|c|c|c|c|c|c|c|}
\hline \multicolumn{7}{|c|}{$m_{\Sigma}=1,157 \mathrm{\kappa \Gamma}(\mathrm{kg}), k_{\mathrm{Ip}}=1,85 \cdot 10^{5} \mathrm{H} / \mathrm{M}(\mathrm{N} / \mathrm{m}), \delta_{0}=5 \cdot 10^{-3} \mathrm{M}(\mathrm{m}), \delta_{\mathrm{cr}}=1 \cdot 10^{-3} \mathrm{M}(\mathrm{m})$} \\
\hline \multirow[t]{2}{*}{$k$} & \multicolumn{2}{|c|}{$\begin{aligned} & R_{\mathrm{Mex}}=10 \mathrm{H} \cdot \mathrm{c} / \mathrm{M} \\
&(\mathrm{N} \cdot \mathrm{s} / \mathrm{m})\end{aligned}$} & \multicolumn{2}{|c|}{$\begin{aligned} R_{\text {Mex }} & =100 \mathrm{H} \cdot \mathrm{c} / \mathrm{M} \\
& (\mathrm{N} \cdot \mathrm{s} / \mathrm{m})\end{aligned}$} & \multicolumn{2}{|c|}{$\begin{array}{c}R_{\text {Mex }}=462,5 \mathrm{H} \cdot \mathrm{c} / \mathrm{M} \\
(\mathrm{N} \cdot \mathrm{s} / \mathrm{m})\end{array}$} \\
\hline & $k V, \%$ & $k V, д Б(\mathrm{~dB})$ & $k V, \%$ & $k V$, дБ $(\mathrm{dB})$ & $k V, \%$ & $k V$, дБ (dB) \\
\hline 0 & 0 & $-\infty$ & 0 & $-\infty$ & 0 & $-\infty$ \\
\hline 1 & 100 & 0 & 100 & 0 & 100 & 0 \\
\hline 2 & 1,44 & 36,83 & 14,44 & $-16,82$ & 68,12 & $-3,33$ \\
\hline 3 & 0,81 & $-41,82$ & 8,15 & $-21,77$ & 42,84 & $-7,36$ \\
\hline 4 & 0,57 & $-44,78$ & 5,8 & $-24,73$ & 31,25 & $-10,1$ \\
\hline 5 & 0,45 & $-46,93$ & 4,53 & $-26,87$ & 24,66 & $-12,16$ \\
\hline 6 & 0,37 & $-48,62$ & 3,73 & $-28,56$ & 20,39 & $-13,81$ \\
\hline 7 & 0,32 & $-50,03$ & 3,17 & $-29,97$ & \begin{tabular}{|l|}
17,39 \\
\end{tabular} & $-15,19$ \\
\hline 8 & 0,27 & $-51,23$ & 2,76 & $-31,17$ & 15,17 & $-16,39$ \\
\hline 9 & 0,24 & $-52,28$ & 2,45 & $-32,22$ & 13,46 & $-17,42$ \\
\hline 10 & 0,22 & $-53,22$ & 2,20 & $-33,15$ & \begin{tabular}{|l|}
12,09 \\
\end{tabular} & $-18,35$ \\
\hline
\end{tabular}

Таблица 6. Алплитудный спектр неосновных гармоник виброскорости ВЭМА от воздействия на механический контур вынуждающей силь прямоугольной форлы при разных величинах коэффициента вязкого трения $\left(R_{\text {Mex } \_ \text {гр }_{1}}=2 \sqrt{k_{\text {пр }} m_{\Sigma}}=925,3 \mathrm{H} \cdot \mathrm{c} / \mathrm{m}\right)$

Table 6. Amplitude spectrum of minor harmonics of vibration electromagnetic activator vibration velocity from the im pact of rectangular driving force on mechanical circuit with varying values of mechanical resistance $\left(R_{\text {мex }_{-} \text {г }}=2 \sqrt{k_{\text {пр }} m_{\Sigma}}=925,3 \mathrm{~N} \cdot \mathrm{s} / \mathrm{m}\right)$

\begin{tabular}{|c|c|c|c|c|c|c|}
\hline \multicolumn{6}{|c|}{$m_{\Sigma}=1,157 \mathrm{\kappa г}(\mathrm{kg}), k_{\text {пр }}=1,85 \cdot 10^{5} \mathrm{H} / \mathrm{M}(\mathrm{N} / \mathrm{m}), \delta_{0}=5 \cdot 10^{-3} \mathrm{M}(\mathrm{m}), \delta_{\text {ст }}=1 \cdot 10^{-3} \mathrm{M}(\mathrm{m})$} \\
\hline \multirow{2}{*}{$k$} & \multicolumn{2}{|c|}{$\begin{array}{r}R_{\text {мех }}=10 \mathrm{H} \cdot \mathrm{c} / \mathrm{M} \\
(\mathrm{N} \cdot \mathrm{s} / \mathrm{m})\end{array}$} & \multicolumn{2}{|c|}{$\begin{array}{r}R_{\text {мех }}=100 \mathrm{H} \cdot \mathrm{c} / \mathrm{M} \\
(\mathrm{N} \cdot \mathrm{s} / \mathrm{m})\end{array}$} & \multicolumn{2}{r|}{$\begin{array}{r}R_{\text {мех }}=462,5 \mathrm{H} \cdot \mathrm{c} / \mathrm{M} \\
(\mathrm{N} \cdot \mathrm{s} / \mathrm{m})\end{array}$} \\
\cline { 2 - 8 } & $A V, \%$ & $A V$, дБ $(\mathrm{dB})$ & $A V, \%$ & $A V$, дБ $(\mathrm{dB})$ & $A V, \%$ & $A V$, дБ $(\mathrm{dB})$ \\
\hline 0 & 0 & $-\infty$ & 0 & $-\infty$ & 0 & $-\infty$ \\
\hline 1 & 100 & 0 & 100 & 0 & 100 & 0 \\
\hline 2 & 0 & $-\infty$ & 0 & $-\infty$ & 0 & $-\infty$ \\
\hline 3 & 0,27 & $-51,37$ & 2,72 & $-31,32$ & 14,28 & $-16,91$ \\
\hline 4 & 0 & $-\infty$ & 0 & $-\infty$ & 0 & $-\infty$ \\
\hline 5 & 0,09 & $-60,91$ & 0,91 & $-40,85$ & 4,93 & $-26,14$ \\
\hline 6 & 0 & $-\infty$ & 0 & $-\infty$ & 0 & $-\infty$ \\
\hline 7 & 0,045 & $-66,93$ & 0,454 & $-46,86$ & 2,48 & $-32,09$ \\
\hline 8 & 0 & $-\infty$ & 0 & $-\infty$ & 0 & $-\infty$ \\
\hline 9 & 0,027 & $-71,37$ & 0,272 & $-51,30$ & 1,49 & $-36,51$ \\
\hline 10 & 0 & $-\infty$ & 0 & $-\infty$ & 0 & $-\infty$ \\
\hline
\end{tabular}

Для увеличения производительности ВЭМА необходим рост резонансной частоты. Этого можно добиться, применив пружину с большей жёсткостью. Увеличение жёсткости приведёт к повышению добротности, увеличению максимальной потенциальной энергии пружины. Поэтому при работе ВЭМА в режиме свободно-вынужденных колебаний произойдёт увеличение силы инерции и амплитуды виброускорения, что положительно скажется на процессах перемешивания и разжижения высоковязких жидкостей.

В ходе аналитических исследований идеальной вынуждающей силы было обнаружено, что она имеет отрицательную составляющую, которую невозможно сформировать в ВЭМА, так как сила является по своей природе только стягивающей и не может быть отталкивающей. Вследствие этого колебание якоря-активатора ВЭМА не может быть строго гармоническим, но можно добиться того, чтобы содержание неосновных гармоник виброскорости было минимальным. Это достигается повышением добротности механического колебательного контура или снижением коэффициента вязкого трения. ВЭМА- 0,3 работает с коэффициентом вязкого трения практически на порядок (в 9,25 раза) меньшим граничного и в силу этого содержание неосновных гармоник виброскорости относительно невысоко (табл. 5,6 ).

При разжижении высоковязких жидкостей с помощью ВЭМА, в первую очередь нефтепродуктов с высоким содержанием парафинов и асфальтенов, величина коэффициента вязкого трения не остаётся постоянной, а уменьшается по ходу работы ВЭМА. Снижение коэффициента вязкого трения колебательного контура приводит к увеличению добротности, максимального зазора магнитной цепи, резонансной частоты и показателя колебательности механического контура, а вследствие возрастания резонансной частоты увеличивается и амплитуда виброускорения, что, в конечном счёте, приводит к повышению эффективность разжижения рабочей высоковязкой жидкости.

Необходимо отметить, что в силу объективных причин механический контур ВЭМА рассматривался упрощенно, но такой анализ позволил обнаружить основные закономерности его работы. В дальнейшем представляется интересным исследовать варианты конструкции ВЭМА с двухполупериодным режимом работы, так как в этом случае представляется возможным получить идеальную вынуждающую колебания силу.

\section{Настройка ВЭМА на режим резонансных колебаний с предельной амплитудой}

Задачами устройства (рис. 7) является автоматическая подстройка частоты и управление амплитудой механических колебаний якоря-активатора BЭMA $[24,25]$. 


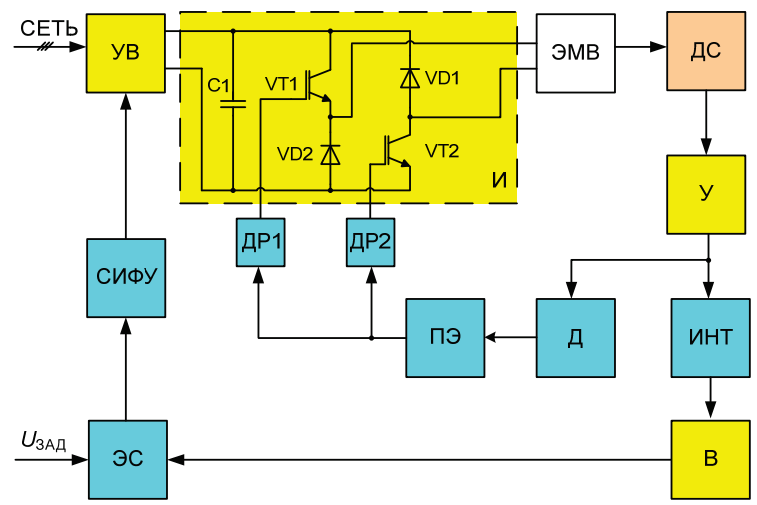

Pис. 7. Функциональная схела устройства, обеспечивающего на стройку ВЭМА на режил резонансных колебаний с предельной амплитудой

Fig. 7. Functional scheme of a device, providing vibration electromagnetic activators regulation for a resonant oscillation mode with a limiting amplitude

Устройство, обеспечивающее настройку ВЭМА на режим резонансных колебаний с предельной амплитудой, работает следующим образом. После включения устройства рабочий орган электромагнитного вибратора (ЭМВ) совершает колебательные движения с частотой собственных колебаний. На выходных зажимах датчика скорости (ДС), усилителя (У) и блока дифференцирования (Д) появляются сигналы синусоидальной формы, частота которых равна частоте колебаний рабочего органа вибратора. Пороговый элемент (ПЭ) преобразует синусоидальный сигнал в прямоугольные управляющие импульсы, положительное значение на входе преобразуется в логическую единицу, отрицательное - в ноль. Далее импульсы управления через драйверы (ДР1, ДР2) поступают на транзисторы инвертора (И). В течение единичного уровня на выходе порогового элемента IGBT транзисторы VT1 и VT2 открыты, что приводит к протеканию тока через обмотку электромагнитного вибратора и притягиванию рабочего органа. В течение нулевого уровня IGBT транзисторы закрыты, а энергия, накопленная в обмотке электромагнитного вибратора, сбрасывается через диоды VD1 и $V D 2$ на конденсатор $C 1$ инвертора (И). Таким образом, реактивная мощность не рассеивается, а используется для формирования следующей полуволны колебаний. Чтобы спадающий в катушке ЭМВ ток не препятствовал движению рабочего органа в обратную сторону, опорный сигнал порогового элемента может быть установлен не нулевым, а небольшим положительным значением. Данная мера несколько уменьшит интервал открытого состояния транзисторов и предоставит дополнительное время для сброса реактивной мощности.

В то же время сигнал, пропорциональный скорости рабочего органа электромагнитного вибрато$\mathrm{pa}$, снимаемый с датчика скорости, усиленный с помощью усилителя и проинтегрированный блоком интегрирования (ИНТ), пропорционален коле- баниям рабочего органа, а сигнал на выходе выпрямителя (В) пропорционален амплитуде этих колебаний. Элемент сравнения (ЭС) сравнивает этот сигнал с сигналом задания $U_{\text {зал, }}$, их разность подается в систему импульсно-фазового управления (СИФУ). СИФУ преобразует постоянное во времени напряжение в импульсы прямоугольной формы, длительность которых зависит от величины входного напряжения. В зависимости от длительности выходных импульсов формирователя меняются углы отпирания тиристоров управляемого выпрямителя (УВ) и тем самым меняются величины напряжений управляемого выпрямителя и инвертора.

Таким образом, устройство управления осуществляет стабилизацию амплитуды колебаний электромагнитного вибратора в резонансном режиме работы.

\section{Оценка погрешности линейной}

\section{математической системы ВЭМА на основе АЧХ}

Выше приведены результаты исследования процессов резонансных колебаний с предельной амплитудой в механической системе вибрационного электромагнитного активатора ВЭМА-0,3 с применением линеаризованной математической модели. Параметры этой модели были идентифицированы на основе решения системы нелинейных алгебраических уравнений, связывающих искомые параметры механической системы с граничными частотами полосы пропускания. Методика подробно изложена в ранее опубликованной работе [26]. На рис. 8 представлены экспериментальные и аналитические АЧХ в двух основных обрабатываемых средах - нефти и воде, при варьировании тока от 1,5 до $5 \mathrm{~A}$.

Так как параметры $R_{\text {мех }}$ и $m_{\Sigma}$ определялись на основе граничных частот полосы пропускания, как и ожидалось, экспериментальные и аналитические АЧХ строго совпадают в двух точках: при нижней $f_{\text {a_m_1 } 1}$ и верхней $f_{\text {a_m__2 }}$ граничных частотах полосы пропускания. Но в силу линеаризации математической модели механической системы, а также из-за особенностей конструкции якоря-активатора ВЭМА резонансные частоты на экспериментальной и аналитической АЧХ несколько различаются (рис. 8). Погрешность линеаризации математической модели механической системы ВЭМА оценим на основе рассогласования резонансных частот как:

$$
\delta f=\frac{\left|f_{0 . \text { эксп }}-f_{\text {0. аналит }}\right|}{f_{\text {оэксп }}} \cdot 100 \%,
$$

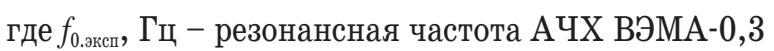
на основе данных эксперимента; $f_{\text {о.валии }}$, Гц - резонансная частоты АЧХ ВЭМА-0,3 на основе аналитических расчетов.

Результаты расчета этой погрешности сведены в табл. 1. 

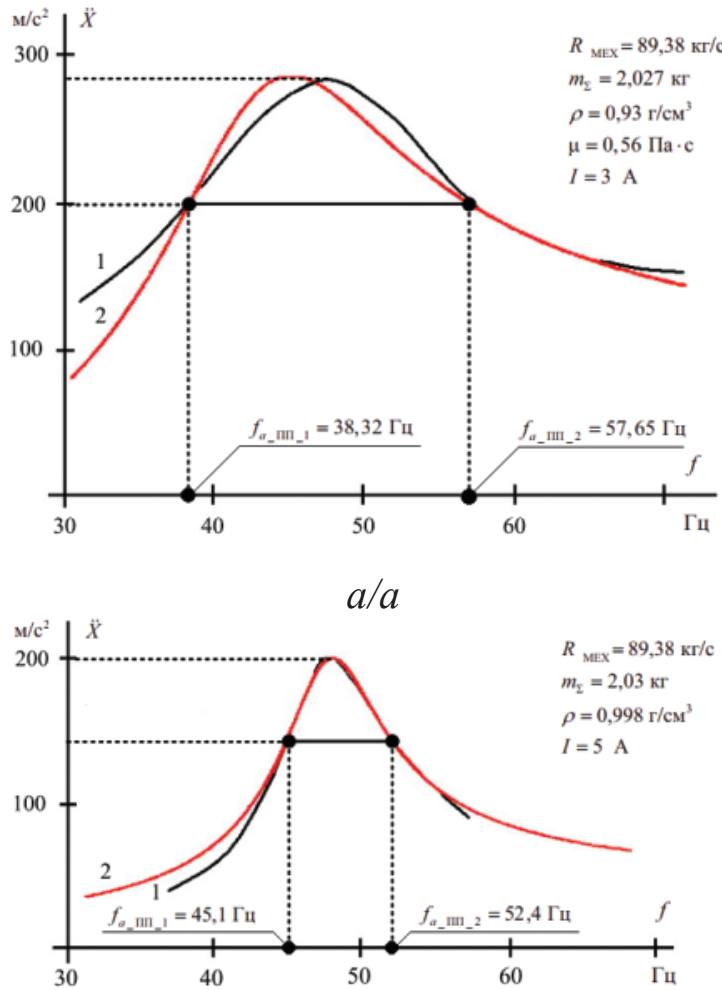

$6 / b$

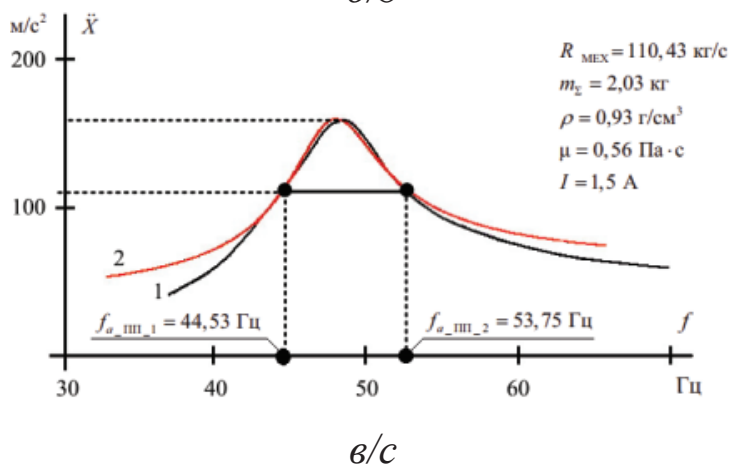

Pис.8. АЧХ: 1 - аналитическая; 2 - экспериментальная; а) нефть; б) вода; в) нефть

Fig. 8. Frequency response; 1 - analytical; 2 - experimental; a) petroleum; b) water; c) petroleum

На основе данных табл. 7 можно отметить, что обобщенная погрешность лежит в пределах от 1,06 до $6,03 \%$. Эта погрешность относительно невысока, что косвенно подтверждает правомочность допущений, принятых выше в данной статье при рассмо-

\section{СПИСОК ЛИТЕРАТУРЫ}

1. Aver'yanov G.S., Khamitov R.N., Zubarev A.V. Dynamics of oscillatory systems with controllable shock absorbers // Russian Engineering Research. - 2008. - V. 28 (6). - P. 543-547.

2. Khamitov R.N., Aver'yanov G.S., Korchagin A.B. Pneumatic shock absorber with an active damping system // Russian Engineering Research. - 2009. - V. 29 (9). - P. 871-873. трении режимов резонансных колебаний с предельной амплитудой в механической системе ВЭМА.

Таблица 7. Результаты расчета погрешности определения резонансной частоты ВЭМА-0.3

Table 7. Results of calculation of the error in determining the resonant frequency VEMA-0.3

\begin{tabular}{|c|c|c|c|}
\hline \multirow{2}{*}{$\begin{array}{c}\text { Параметры } \\
\text { Parameters }\end{array}$} & \multicolumn{3}{|c|}{$\begin{array}{r}\text { Условия проведения и результаты эксперимента } \\
\text { Conditions and results of the experiment }\end{array}$} \\
\cline { 2 - 4 } & Нефть/Petroleum & Вода Water & Нефть/Petroleum \\
\hline I, А (A) & 3,0 & 5,0 & 1,5 \\
\hline$f_{\text {а_пп_1 }}$, Гц $(\mathrm{Hz})$ & 38,22 & 45,1 & 44,53 \\
\hline$f_{\text {а_пп_2 }}$, Гц $(\mathrm{Hz})$ & 57,65 & 52,4 & 53,75 \\
\hline$R_{\text {мех }}$, кГ $/ \mathrm{c}(\mathrm{kg} / \mathrm{s})$ & 232,71 & 89,38 & 110,43 \\
\hline$m_{\Sigma}$, кг $(\mathrm{kg})$ & 2,447 & 2,027 & 2,026 \\
\hline$f_{0 . \text { эксп }}$, Гц $(\mathrm{Hz})$ & 45,09 & 48,06 & 47,97 \\
\hline$f_{0 . \text { аналит }}$, Гц $(\mathrm{Hz})$ & 47,81 & 47,55 & 48,59 \\
\hline$\delta f, \%$ & 6,03 & 1,06 & 1,29 \\
\hline \multicolumn{4}{|c|}{} \\
\hline
\end{tabular}

\section{Выводы}

1. На основании обзора источников показана эффективность и перспективность применения вибрационных электромагнитных активаторов для подготовки буровых растворов и разжижения высоковязких нефтепродуктов.

2. Предложен оригинальный подход для исследования резонансных колебаний с предельной амплитудой в механической системе вибрационных электромагнитных активаторов на основе линейной математической модели. Исследован режим свободных колебаний якоря-активатора с предельной амплитудой в первом полупериоде резонансных колебаний при вариации параметров механической системы, что обусловлено изменением физических свойств обрабатываемых жидких сред. Получено аналитическое выражение для вынуждающей силы, обеспечивающей режим вынужденных колебаний, который предлагается рассматривать как естественное дополнение режима свободных колебаний.

3. Исследовано разработанное техническое решение, обеспечивающее настройку вибрационных электромагнитных активаторов на режим резонансных колебаний с предельной амплитудой.

4. На основе сопоставительного анализа экспериментальных и модельных частотных характеристик продемонстрирован приемлемый уровень погрешности применения линейной математической модели механической системы $1,06 . .6,03 \%$ при настройке ВЭМА на режим резонансных колебаний с предельной амплитудой.

3. Pevchev V.P. The superexitation and efficiency relation in a short-stroke pulsed electromagnetic motor of a seismic source // Journal of Mining Science. - 2010. - V. 46. - № 6. - P. 656-665.

4. Кувшинов К.А., Мойзес Б.Б., Крауиньш П.Я. Импульсно-вибрационный источник сейсмических сигналов // Известия Томского политехнического университета. - 2010. - Т. 317. № 1. - C. 77-81. 
5. Simonov B.F., Neiman V.Y., Shabanov A.S. Pulsed Linear Solenoid Actuator for Deep-Well Vibration Source // Journal of Mining Science. - 2017. - V. 53 (1). - P. 117-125.

6. Шнеерсон М.Б. Теория и практика наземной сейсморазведки. - М.: Недра, 1988. - 527 с.

7. Pevchev V.P. Science of mining machines the superexcitation and efficiency relation in a short-Stroke pulsed electromagnetic motor of a seismic source // Journal of Mining Science. - 2010. V. $46(6) .-$ P. $656-665$.

8. Нейман Л.А., Нейман В.Ю. Моделирование динамических процессов в электромагнитных преобразователях энергии для систем генерирования силовых воздействий и низкочастотных вибраций // Известия Томского политехнического университета. Инжиниринг георесурсов. - 2015. - Т. 326. - № 4. C. 154-162.

9. Pevchev V.P. Principal dimensions of the short-stroke electromagnetic motor for a seismic wave generator // Journal of Mining Science. - 2009. - V. 45. - № 4. - P. 372-381.

10. Oil recovery from tank bottom sludge using rhamnolipids / Chuhan Liu, Yin Zhang, Shanshan Sun, Lixin Huang, Li Yu, Xiaonan Liu, Ruiqiu Lai, Yijing Luo, Zhiyong Zhang, Zhongzhi Zhang // Journal of Petroleum Science and Engineering. 2018. - V. 170. - P. 14-20.

11. Analysis of oil content in drying petroleum sludge of tank bottom / Yuhua Wang, Xiaomin Zhang, Yuying Pan, Ying Chen // International Journal of Hydrogen Energy. - 2017. -V. 42. - № 29. P. 18681-18684.

12. Da S.L., Alves F.C., De França F.P. A review of the technological solutions for the treatment of oily sludges from petroleum refineries // Waste Management \& Research. - 2012. - V. 30 (10). P. $1016-1030$.

13. Исследование процесса струйного размыва донных отложений в нефтяных резервуарах / В.Г. Бутов, А.В. Никульчиков, В.К. Никульчиков, В.А. Солоненков, А.А. Ящук // Известия Томского политехнического университета. Инжиниринг георесурсов. -2018 . - Т. 329. - № 9. - С. 93-100.

14. Nekrasov V.0. Modeling bottom sediment erosion process by swirling the flow by tangential supply of oil in the tank // IOP Conference Series: Materials Science and Engineering: Transport and Storage of Hydrocarbons. - 2016. - V. 154. - 7 p. URL: http://iopscience.iop.org/article/10.1088/1757-899X/ 154/1/012003 (дата обращения 13.06.2018).

15. Burkov P.V., Burkova S.P., Samigullin V.D. Computer Simulation of Stress Strain State of Oil Gathering Pipeline Designed for Ugut Field // IOP Conference Series: Materials Science and Engineering. - 2016. - V. 125: Materials Treatment: Current Problems and Solutions. $-7 \mathrm{p}$.
16. Расчет гидравлических сопротивлений затопленного вибрирующего конфузора электромагнитного вибратора / А.В. Азин, Е.П. Богданов, С.В. Пономарев, С.В. Рикконен // Известия Томского политехнического университета. Инжиниринг георесурсов. - 2017. - Т. 328. - № 2. - С. 67-75.

17. Расчет энергетических параметров затопленного вибрирующего конфузора электромагнитного вибратора / А.В. Азин, Е.П. Богданов, С.В. Пономарев, С.В. Рикконен // Известия Томского политехнического университета. Инжиниринг георесурсов. - 2017. - Т. 328. - № 5. - С. 16-23.

18. Bogdanov E., Nomokonova Y., Rikkonen S. Oscillatory system of the jet electromagnetic vibrator // IOP Conference Series: Materials Science and Engineering. - 2014. - V. 66 (1). - P. 012017.

19. Real time prediction of suspended solids in drilling fluids. Review Article / S.C. Magalhnes, C.M. Scheid, L.A. CalHada, L.M.M. Lutterbach, R.S. Rezende, A.T.A. Waldmann // Journal of Natural Gas Science and Engineering. - March 2016. - V. 30. P. $164-175$.

20. Данекер В.А., Рикконен С.В. Приготовление и коррекция показателей бурового раствора технологией и оборудованием BCMA // Известия Томского политехнического университета. Инжиниринг георесурсов. - 2017. - Т. 328. - № 7. - С. 86-92.

21. Neyman L.A., Neyman V.Y. Dynamic model of a vibratory electromechanical system with spring linkage // Proceedings$201611^{\text {th }}$ International Forum on Strategic Technology, IFOST 2016. - 2017. - № 7884234. - P. 23-27.

22. Нейман Л.А., Нейман В.Ю. Влияние механических потерь на выходные показатели электромеханической колебательной системы с электромагнитным возбуждением // Актуальные проблемы в машиностроении. - 2016. - № 3. - С. 234-240.

23. Neyman L.A., Neyman V.Y., Shabanov A.S. Vibration dynamics of an electromagnetic drive with a half-period rectifier // International Conference of Young Specialists on Micro/Nanotechnologies and Electron Devices, EDM. - 2017. - № 7981805. - P. 503-506.

24. Глазырин А.С. Системы питания и автоматического управления вибрационными электромагнитными активаторами: дисс.... канд. техн. наук. - Томск, 2004. - 193 с.

25. Устройство для управления электромагнитными вибраторами: пат. № 2348961 РФ. МПК G05D19/02 (2006.01). Заявка № 2008102340/28; опубл. 10.03.2008, Бюл. № 7. - 6 с.

26. Идентификация параметров механической системы на примере вибрационного электромеханического преобразователя энергии / А.С. Глазырин, В.В. Тимошкин, С.В. Цурпал, Т.А. Глазырина // Известия Томского политехнического университета. - 2010. - Т. 316. - № 4. - С. 174-177.

Поступила 10.10.2018 2.

\section{Информация об авторах}

Гаврилин А.Н., кандидат технических наук, доцент отделения материаловедения Инженерной школы новых производственных технологий Национального исследовательского Томского политехнического университета.

Боловин $\boldsymbol{E}$.B., ассистент отделения электроэнергетики и электротехники Инженерной школы энергетики Национального исследовательского Томского политехнического университета.

Глазырин A.C., доктор технических наук, доцент отделения электроэнергетики и электротехники Инженерной школы энергетики Национального исследовательского Томского политехнического университета.

$\boldsymbol{K} л а д и е \boldsymbol{C} \boldsymbol{C} \boldsymbol{H} .$, кандидат технических наук, доцент отделения электроэнергетики и электротехники Инженерной школы энергетики Национального исследовательского Томского политехнического университета.

Полищук В.И., доктор технических наук, профессор кафедры электроснабжения промышленных предприятий Алтайского государственного технического университета им. И.И. Ползунова. 


\title{
RESONANT OSCILLATIONS WITH A LIMITING AMPLITUDE IN A VIBRATION ELECTROMAGNETIC ACTIVATOR
}

\author{
Alexey N. Gavrilin', \\ tom-gawral@list.ru \\ Evgeniy V. Bolovin', \\ boloinev@mail.ru
}

\author{
Alexander S. Glazyrin', \\ asglazyrin@tpu.ru
}

\author{
Sergey N. Kladiev', \\ kladiev@tpu.ru

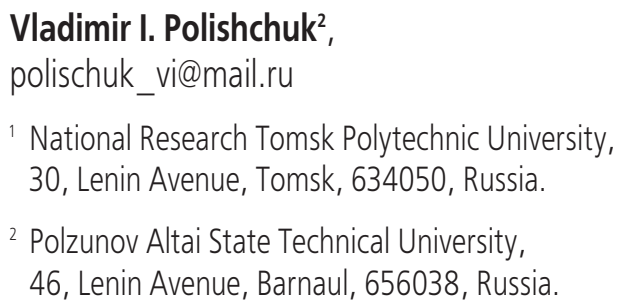

The relevance of the research is caused by the fact that vibration electromagnetic activators are promising for use in various technologies. These technologies are associated with extraction and transportation of georesources, including preparation of drilling fluids and liquefaction of viscous oil products. Vibrating electromagnetic activator is an electric machine of reciprocating motion with an armatureactivator, which forms turbulent submerged jets in a treated liquid medium. In the first half-period, the armature-activator is attracted to the wall due to a current pulse in the coils and potential energy is accumulated in the elastic spring. This is a mode of forced oscillations in mechanical system of a vibrating electromagnetic activator. In the second half-period there is no current in the coils and the armature-activator is repelled from the walls due to the spring energy. This is the mode of free oscillations in the mechanical system. Vibration electromagnetic activators should be tuned to the resonant frequency to maximize energy efficiency. The resonant frequency is not constant and depends on the properties of the treated fluid. The shape of the force-induced oscillation must provide oscillations in mechanical system at the resonant frequency with a limiting amplitude. This fact will increase the energy efficiency and performance of a vibration electromagnetic activator.

The main aim of the research is to conduct analytical research to provide resonant oscillations with a limiting amplitude in a vibration electromagnetic activator.

Methods of the research are based on using ordinary differential equations, Laplace transform, amplitude-frequency characteristics, systems of nonlinear algebraic equations, spectral analysis, comparison of analytical and experimental characteristics

Results. The authors have considered the mode of free oscillations of the armature-activator with the limiting amplitude based on linearized mathematical model of the mechanical vibration electromagnetic activator system. The mode of forced oscillations is proposed to be considered as a natural addition to the mode of free oscillations with a driving force that acts half the period and has a special form. It is shown that the modes of free and forced oscillations substantially depend on the parameters of the mechanical system. Such parameters depend on the properties of the treated liquid medium. The authors carried out the spectral analysis of the driving force, providing resonant oscillations with a limiting amplitude in the vibration electromagnetic activator mechanical system. The paper introduces the technical solution that ensures the control of the vibration electromagnetic activator with automatic tuning to the resonant frequency and the limiting amplitude of oscillations of the armature-activator. This mode maximizes the energy efficiency and productivity of the mixing liquid processed media. Based on a comparison of analytical and experimental frequency characteristics the authors confirmed the hypothesis of admissibility of linearizing the mathematical model of vibration electromagnetic activator mechanical system in an analytical study of the driving force of the optimal form.

\section{Key words:}

Drilling fluid, highly viscous oil product, vibratory electromagnetic activator, resonance, force-induced oscillations, free oscillations, forced oscillations, spectral analysis, tuning to the resonant frequency, error estimate.

\section{REFERENCES}

1. Aver'yanov G.S., Khamitov R.N., Zubarev A.V. Dynamics of oscillatory systems with controllable shock absorbers. Russian Engineering Research, 2008, vol. 28 (6), pp. 543-547.

2. Khamitov R.N., Aver'yanov G.S., Korchagin A.B. Pneumatic shock absorber with an active damping system. Russian Engine ering Research, 2009, vol. 29 (9), pp. 871-873.
3. Pevchev V.P. The superexitation and efficiency relation in a short-stroke pulsed electromagnetic motor of a seismic source. Journal of Mining Science, 2010, vol. 46, no. 6, pp. 656-665.

4. Kuvshinov K.A., Moizes B.B., Krauinsh P.Ya. Pulse-vibration source of seismic signalsю Bulletin of the Tomsk Polytechnic University, 2010, vol. 317, no. 1, pp. 77-81. In Rus. 
5. Simonov B.F., Neiman V.Y., Shabanov A.S. Pulsed Linear Solenoid Actuator for Deep-Well Vibration Source. Journal of Mining Science, 2017, vol. 53 (1), pp. 117-125.

6. Shneerson M.B. Teoriya i praktika nazemnoy seysmorazvedki [Theory and practice of ground seismic exploration]. Moscow, Nedra Publ., 1988. 527 p.

7. Pevchev V.P. Science of mining machines the superexcitation and efficiency relation in a short-Stroke pulsed electromagnetic motor of a seismic source. Journal of Mining Science, 2010, vol. 46 (6), pp. 656-665.

8. Neyman L.A., Neyman V.U. Simulation of dynamic processes in electromagnetic energy converters for force effects and lowfrequency vibrations generation systems. Bulletin of the Tomsk Polytechnic University, 2015, vol. 326, no. 4, pp. 154-162. In Rus.

9. Pevchev V.P. Principal dimensions of the short-stroke electromagnetic motor for a seismic wave generator. Journal of Mining Science, 2009, vol. 45, no. 4, pp. 372-381.

10. Chuhan Liu, Yin Zhang, Shanshan Sun, Lixin Huang, Li Yu, Xiaonan Liu, Ruiqiu Lai, Yijing Luo, Zhiyong Zhang, Zhongzhi Zhang. Oil recovery from tank bottom sludge using rhamnolipids. Journal of Petroleum Science and Engineering, 2018, vol. 170, pp. 14-20.

11. Yuhua Wang, Xiaomin Zhang, Yuying Pan, Ying Chen. Analysis of oil content in drying petroleum sludge of tank bottom. International Journal of Hydrogen Energy, 2017, vol. 42, no. 29, pp. 18681-18684.

12. Da S.L., Alves F.C., De França F.P. A review of the technological solutions for the treatment of oily sludges from petroleum refineries. Waste Management \& Research, 2012, vol. 30 (10), pp. 1016-1030.

13. Butov V.G., Nikulchikov A.V., Nikulchikov V.K., Solonenko V.A., Yashchuk A.A. Simulation study of bottom sediments jet erosion in oil tank. Bulletin of the Tomsk Polytechnic University. Geo assets engineering, 2018, vol. 329, no. 9, pp. 93-100. In Rus.

14. Nekrasov V.0. Modeling bottom sediment erosion process by swirling the flow by tangential supply of oil in the tank. IOP Conference Series: Materials Science and Engineering: Transport and Storage of Hydrocarbons, 2016, vol. 154, $7 \mathrm{p}$.

15. Burkov P.V., Burkova S.P., Samigullin V.D. Computer Simulation of Stress Strain State of Oil Gathering Pipeline Designed for Ugut Field. IOP Conference Series: Materials Science and Engineering, 2016, vol. 125, $7 \mathrm{p}$.

16. Azin A.V., Bogdanov E.P., Ponomarev S.V., Rikkonen S.V. Calculation of hydraulic resistances of a submerged vibrating con- fuser of an electromagnetic vibrator. Bulletin of the Tomsk Polytechnic University. Geo Assets Engineering, 2017. vol. 328, no. 2, pp. $67-75$. In Rus.

17. Azin A.V., Bogdanov E.P., Ponomarev S.V., Rikkonen S.V. Calculation of energy parameters of submerged vibrating confuser of an electromagnetic vibrator. Bulletin of the Tomsk Polytechnic University. Geo Assets Engineering, 2017, vol. 328, no. 5, pp. 16-23.

18. Bogdanov E., Nomokonova Y., Rikkonen S. Oscillatory system of the jet electromagnetic vibrator. IOP Conference Series: Materials Science and Engineering, 2014, vol. 66 (1), pp. 12-17.

19. Magalhnes S.C., Scheid C.M., CalHada L.A., Lutterbach L.M.M., Rezende R.S., Waldmann A.T.A.. Real time prediction of suspended solids in drilling fluids. Review Article. Journal of Natural Gas Science and Engineering, March 2016, vol. 30, pp. 164-175.

20. Daneker V.A., Rikkonen S.V. Preparation and correction of drilling mud by technology and equipment of vibro-jet-magnetic activation. Bulletin of the Tomsk Polytechnic University. Geo Assets Engineering, 2017, vol. 328, no. 7, pp. 86-92. In Rus.

21. Neyman L.A., Neyman V.Y. Dynamic model of a vibratory electromechanical system with spring linkage. $11^{\text {th }}$ International $\mathrm{F}_{0}$ rum on Strategic Technology, 2017, no. 7884234, pp. 23-27.

22. Neyman L.A., Neyman V.Yu. Vliyanie mekhanicheskikh poter na vykhodnye pokazateli elektromekhanicheskoy kolebatelnoy sistemy s elektromagnitnym vozbuzhdeniem [The effect of mechanical losses on the output indicators of an electromechanical oscillatory system with electromagnetic excitation]. Aktualnye problemy $v$ mashinostroenii, 2016, no. 3, pp. 234-240.

23. Neyman L.A., Neyman V.Y., Shabanov A.S. Vibration dynamics of an electromagnetic drive with a half-period rectifier. International Conference of Young Specialists on Micro/Nanotechnologies and Electron Devices, EDM, 2017, no. 7981805, pp. 503-506.

24. Glazyrin A.S. Sistemy pitaniya i avtomaticheskogo upravleniya vibratsionnymi elektromagnitnymi aktivatorami. Diss. Kand. nauk [Power systems and automatic control of vibration electromagnetic activators. Cand. Sc. Diss.]. Tomsk, 2004. 193 p.

25. Tsurpal S.V., Glazyrin A.S. Ustroystvo dlya upravleniya elektromagnitnymi vibratorami [Device for controlling electromagnetic vibrators]. Patent RF, no. 2008102340/28, 2008.

26. Glazyrin A.S., Timoshkin V.V., Tsurpal S.V., Glazyrina T.A. Identification of a mechanical system parameters on the example of a vibratory electromechanical energy converter. Bulletin of the Tomsk Polytechnic University, 2010, vol. 316, no. 4, pp. 174-177. In Rus.

\section{Information about the authors}

Alexey N. Gavrilin, Cand. Sc., associate professor, National Research Tomsk Polytechnic University.

Evgeniy V. Bolovin, assistant, National Research Tomsk Polytechnic University.

Alexander S. Glazyrin, Dr. Sc., associate professor, National Research Tomsk Polytechnic University.

Sergey N. Kladiev, Cand. Sc., associate professor, National Research Tomsk Polytechnic University.

Vladimir I. Polishchuk, Dr. Sc., professor, Polzunov Altai State Technical University. 